\title{
Colour Constancy Algorithm Based on the Minimization of the Distance between Colour Histograms ${ }^{\star}$
}

\author{
Jaume Vergés-Llahí and Alberto Sanfeliu \\ Institut de Robòtica i Informàtica Industrial \\ Technological Park of Barcelona, U Building \\ Llorens i Artigas 4-6 2nd Floor, 08028 Barcelona, Spain. \\ \{jverges, asanfeliu\}@iri.upc.es
}

\begin{abstract}
Colour is an important clue in many applications in machine vision and image processing. Despite of this, the drawback of colour is its dependence upon illumination changes. Colour constancy aims to provide colour appearance of objects with stability. This paper presents a simple and robust colour constancy algorithm based on a coefficient transformation of the colour coordinates of pixels which goal is to reduce the distance between histograms of two images of similar scenes under different illuminations. Our main contribution is that our algorithm only make use of raw image data contrary to most usual colour constancy algorithms. We show that our approach is able to cope with colour change since it substantially reduces the distance between colour histograms.

Keywords: colour, colour change, colour constancy, colour histograms.
\end{abstract}

\section{Introduction}

In a number of applications from machine vision tasks such as object recognition, image indexing and retrieval, to digital photography or new multimedia applications, it is important that colours recorded by a device remain constant under changes in scene illumination.

Hence, a preliminary step when using colour must be to remove the pernicious effect of illumination change. This problem is usually referred in literature as colour constancy, i.e., the stability of surface colour appearance under varying illumination conditions.

A great effort has been put lately in solving this problem. Part of the difficulty is due to the fact that this problem is entangled with other confounding phenomena such as the shape of the objects, viewing and illumination geometry besides changes in illumination spectral power distribution and reflectance properties of the imaged objects.

Let us sketch some of the most important approaches and their assumptions. Land [1] assumes that every image contains a white patch. Another assumption

* Partially funded by a fellowship of the Government of Catalonia and the CICYT DPI2001-2223. 
[2] is that the average reflectance of all surfaces in a scene is achromatic, i.e., gray. In this case, the average colour of the light leaving the surface will be the colour of the incident illumination.

Other authors have tried to exploit features not present in the idealised Mondrian world, such as specularities [3], shadows [4], or mutual illumination [5], to recover information about the scene illumination. As reported in [6], the main drawback of all these algorithms is that their assumptions are often violated in regular images. In the other hand, among the others which work on real images, their performance is still far from being good enough as reported in [7].

An alternative is the set of algorithms which do not try to seek for a unique solution, rather the most likely one. For example, the gamut-mapping algorithm developed by Forsyth [8] and expanded later by Finlayson [9, 10] selects a solution from the set of all feasible solutions using different selection criteria.

Other authors $[11,12]$ have posed the problem in a probabilistic framework and, more recently, Sapiro [12] has developed an algorithm based on the probabilistic Hough transform while Finlayson has used this framework in his colour by correlation algorithm [6]. The neural network approach [13] to colour constancy can similarly be seen as a method of dealing with the inherent uncertainty of the problem. All these algorithms represent an improvement at solving the colour constancy problem, but some further work must still be done to find a definitive solution [6].

The main problems in colour constancy algorithms are that they should be solely based upon image data rather than on a set of stored data about illuminants which is most of the times difficult to gather or can be inaccurate. Moreover, some approaches using colour, such as image indexing and retrieval, rely enormously on histograms as a mean of describing colour distributions and comparing the appearance of images. Therefore, it seems clear that histogram distance can be a good tool to lead the search for a proper colour transformation which keeps colour as constant as possible.

This paper describes a simple and robust procedure which consists in minimising an objective function using a downhill simplex minimization scheme. The objective function gauges the dissimilarities in colour of two images by means of the difference of their colour histograms. The minimization is performed in the space of colour transformations. We used the kind of colour transformations habitual in $2 D$ gamut-mapping algorithms, [8-10], which is based on an independent coefficient modifying each colour channel besides of using chromaticity coordinates.

With regard to algorithms such as gamut-mapping [10] or colour by correlation [6], our main contributions consist both in proposing a computational scheme to colour constancy which is completely based on raw image data with no further assumptions about illumination and that of simplifying colour constancy up to a minimization problem driven by a colour measure widely spread in tasks such as image indexing. This way, despite the good performances of the aforementioned approaches, our algorithm also reaches satisfactory results with far fewer a priori information under a varied set of illumination. 


\section{Colour Change Model}

Let be $I^{1}$ and $I^{2}$ two colour images of nearly the same scene taken under different illumination conditions. We take $I^{2}$ as the reference and our goal will be to find a way to change the colour of pixels of the image $I^{1}$ so as to turn them as close as possible to those of image $I^{2}$, i.e., to keep the colour of the scene constant.

Since some features such as shape and shading only affect the magnitude of the reflected light but not its colour the use of the chromaticity coordinates $(r, g)$ is justified. In the present work, we use the chromaticities defined in [6]:

$$
\left(r_{i}, g_{i}\right)=\left(\frac{R_{i}}{B_{i}}, \frac{G_{i}}{B_{i}}\right), i=1, \ldots, N
$$

where $\left(R_{i}, G_{i}, B_{i}\right)$ is the colour response at the $i^{\text {th }}$ pixel and $N$ is the number of pixels in an image.

Our colour constancy algorithm is based on computing a transformation of the following form:

$$
\left(r_{i}^{\prime}, g_{i}^{\prime}\right)=\left(\alpha \cdot r_{i}, \beta \cdot g_{i}\right), i=1, \ldots, N
$$

where $(\alpha, \beta)$ are the coefficients that change coordinates $r_{i}$ and $g_{i}$ into $r_{i}^{\prime}$ and $g_{i}^{\prime}$, respectively. This way, we can denote the transformed imaged from $I$ as $\left.I\right|_{(\alpha, \beta)}$. This sort of transformation has been successfully used in the 2D gamut mapping algorithm of Finlayson $[9,10,6]$ and is based upon the fact that the sensitivity functions of the set of sensors are independent one another.

Provided that the previous model copes with colour change in an adequate way, we now face the problem of computing a proper transformation $\left(\alpha_{0}, \beta_{0}\right)$ out of image data. The main idea followed along this work is that from the space of all feasible transformations $\mathcal{T}$ we must take the one that changes colours in image $I^{1}$ in a way that most resemble those of the reference $I^{2}$. We use the SwainËBallard distance [14] as a mean of computing the colour difference between images $I^{1}$ and $I^{2}$ :

$$
\operatorname{dist}\left(\mathcal{H}^{1}, \mathcal{H}^{2}\right)=\sum_{k} \min \left\{H_{k}^{1}, H_{k}^{2}\right\} \in[0,1]
$$

where $\mathcal{H}^{1}$ and $\mathcal{H}^{2}$ are the chromaticity histograms of images $I^{1}$ and $I^{2}$, respectively. These histograms take into account of colours for each image.

Consequently, we can now define an objective function $f$ which is dependent on $(\alpha, \beta)$ as follows:

$$
f(\alpha, \beta)=\operatorname{dist}\left(\left.\mathcal{H}^{1}\right|_{(\alpha, \beta)}, \mathcal{H}^{2}\right)
$$

where $\left.\mathcal{H}^{1}\right|_{(\alpha, \beta)}$ is the histogram of the transformed image $\left.I^{1}\right|_{(\alpha, \beta)}$ and $\mathcal{H}^{2}$ that of the reference $I^{2}$.

This way, the problem of colour constancy has been translated into a problem of minimization, i.e., that of finding the transformation $\left(\alpha_{0}, \beta_{0}\right)$ such that:

$$
\left(\alpha_{0}, \beta_{0}\right)=\underset{(\alpha, \beta) \in \mathcal{T}}{\operatorname{argmin}}\{f(\alpha, \beta)\}
$$


Once we have a transformation $\left(\alpha_{0}, \beta_{0}\right)$, we can recover the true colours of $I^{1}$ from the transformed image $\left.I^{1}\right|_{\left(\alpha_{0}, \beta_{0}\right)}$ which colours are close to those of image $I^{2}$ in the following way:

$$
\left(R_{i}^{\prime}, G_{i}^{\prime}, B_{i}^{\prime}\right)=\left(r_{i}^{\prime} \cdot B_{i}, g_{i}^{\prime} \cdot B_{i}, 1 \cdot B_{i}\right), i=1, \ldots, N
$$

where $\left(R_{i}^{\prime}, G_{i}^{\prime}, B_{i}^{\prime}\right)$ forms the $i^{t h}$ pixel of the image $\left.I^{1}\right|_{\left(\alpha_{0}, \beta_{0}\right)}$.

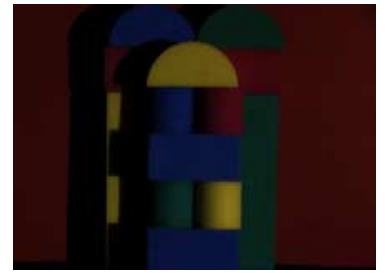

I1: Solux 3500

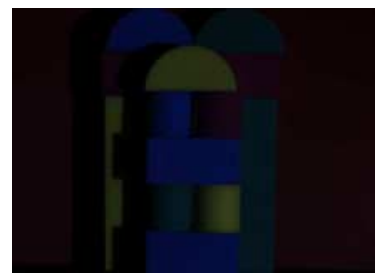

I4: Solux 3500+3202

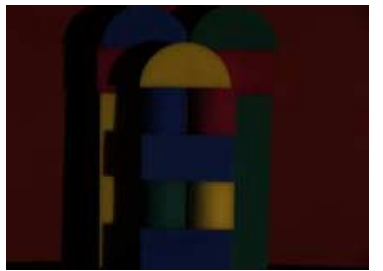

I7: Syl. 50MR

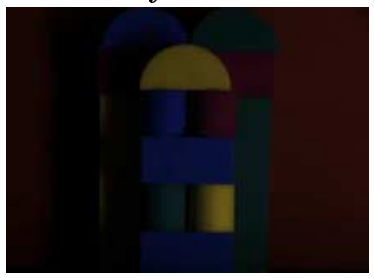

I10: Sylvania CWF

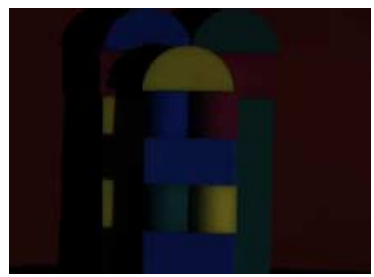

I2: Solux 4100

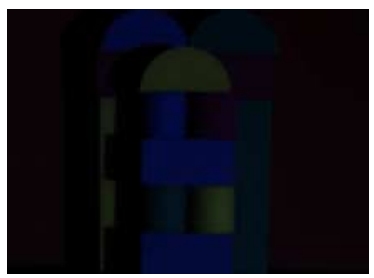

I5: Solux $4100+3202$

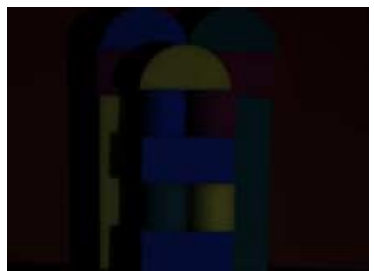

I8: Syl. 50MR+3202

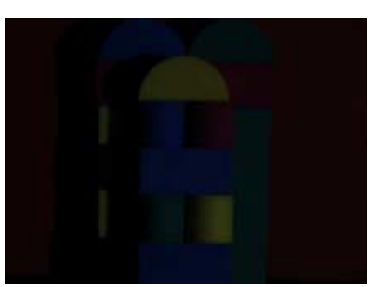

I3: Solux 4700

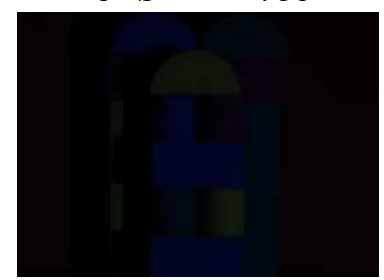

I6: Solux $4700+3202$

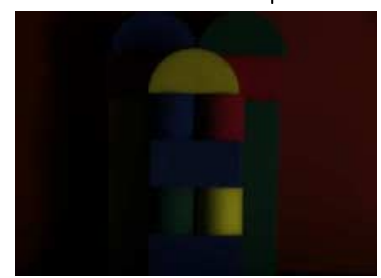

I9: Philips UF

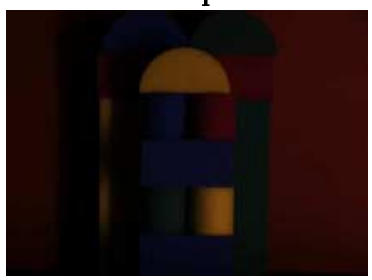

I11: Sylvania WWF

Fig. 1. Set of images. 


\section{Downhill Simplex Minimization Algorithm}

The problem of minimization consists, once a function $f$ depending on one or more independent variables is given, in finding the value of those variables where $f$ takes on a maximum or a minimum value.

Moreover, some methods need to evaluate the derivatives of the function. Despite the fact that algorithms with derivatives are more powerful than those using only the function, they are sometimes computationally expensive or numerically unstable and does not always compensate for the additional calculations.

Due to the precise nature of our problem, where computing the objective function is computationally expensive and derivatives are somewhat unstable because of the discretisation of histograms, it has been clear at once to use of a minimization method which needs no evaluations of the derivatives of the objective function.

The downhill simplex method due to Nelder and Mead [15] requires only function evaluations, not derivatives. This method crawls downhill in a straightforward way that makes almost no special assumption about the objective function. It can be argued that it is not the most efficient algorithm in terms of the required number of function evaluations what other methods [15] could almost surely outperform. However, downhill simplex method is extremely robust and needs far lesser information.

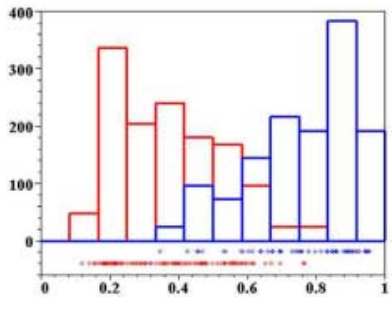

Colour distances.

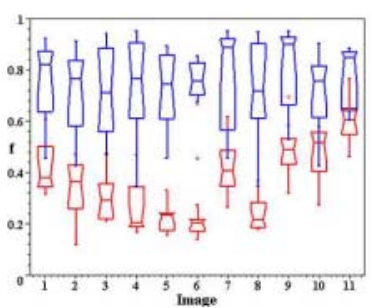

Results per image.

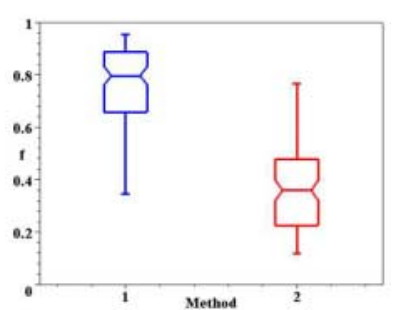

Global Results.

Fig. 2. Colour constancy results.

A simplex is the geometrical figure consisting, in $M$ dimensions, of $M+1$ points (vertices) and all their interconnecting segments, faces, etc. In $2 D$, a simplex is a triangle; in $3 D$, it is a tetrahedron.

The goal of the downhill simplex method is to transform an initial guess simplex by means of a set of moves (reflection, contraction and expansion) of its vertices so as to embrace a valley. Then the simplex is contracted pulling itself in around the lowest (best) point. As any multidimensional minimization routine, a termination criteria must be given, which can be either the decrease in the function value or the distance the point moved in a step.

As an initial simplex we try the triangle $\mathcal{T}=\left\{P_{0}, P_{0}+(\lambda, 0), P_{0}+(0, \lambda)\right\}$, where $P_{0}$ is the starting point and $\lambda$ is a constant for the problem's length scale. 
Thus, the point $\left(\alpha_{0}, \beta_{0}\right) \in \mathcal{T}$ found is a (local, at least) minimum of the objective functions $f$ and represents the colour transformation for colour constancy. To likely find a point close to a global minimum, we perform the search at different starting points $P_{0}$ of the transformation space and take the better one.

\section{Results}

In this section, we perform the previous algorithm in a set of 11 images of a scene taken under different illuminations. We compare the algorithm results with the worst performance obtained when no colour constancy algorithm is carried out.

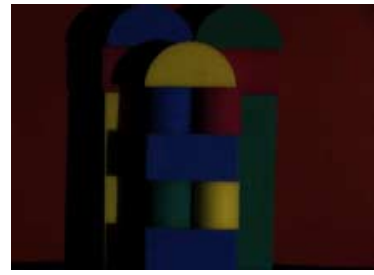

Ref. (I1)

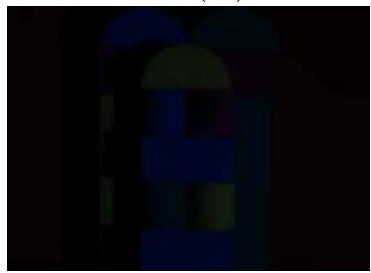

Ref. (I6)

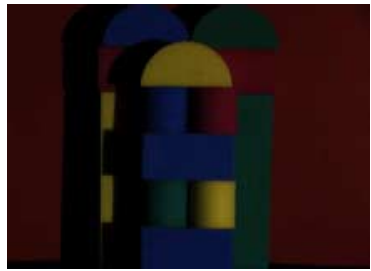

Ref. (I1)

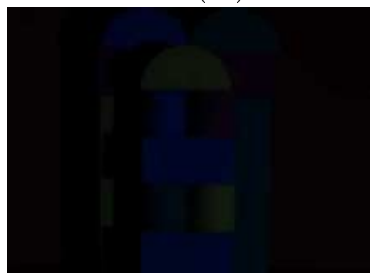

Ref. (I6)

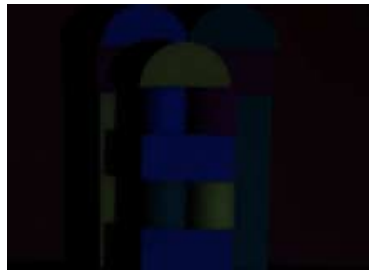

Test (I5)

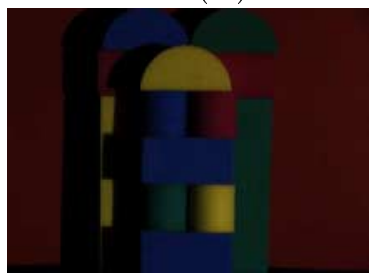

Test (I1)

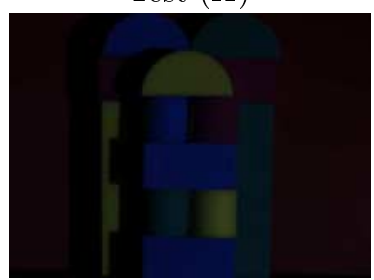

Test (I3)

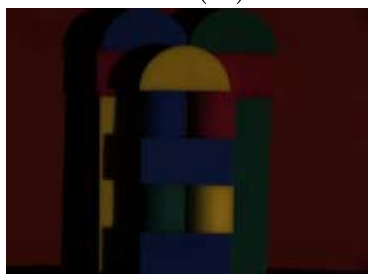

Test (I7)

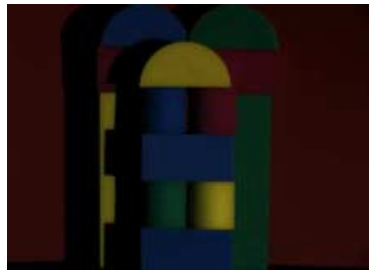

Result

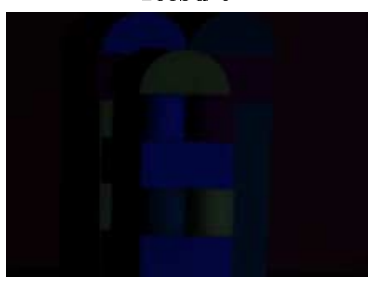

Result

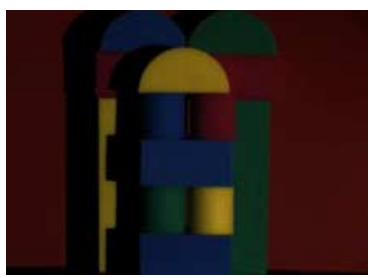

Result

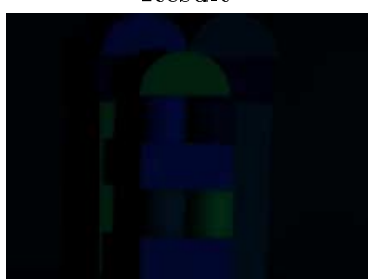

Result

Fig. 3. Some resultant images. 
The objects displayed in these images are real block figures of different plain colours. In Fig. 1 we show the set of images. This set belongs to the database of the Computational Vision Lab at the Simon Fraser University and can be found via Internet at the following URL in http://www.cs.sfu.ca/ colour/.

Objects present shading due to their volumes and shadows because of the direction of the incident light. The nature of the illumination that were used to acquire the images are both bulb and fluorescent lights. Various kinds of daylight bulbs were taken into account, each of different colour temperature $(3500 \mathrm{~K}, 4100 \mathrm{~K}$ and $4700 \mathrm{~K})$, as well as a mixture of them.

The experiment consists in taking an image as a reference while computing a colour transformation for the rest of images onto the reference. Afterwards, we compare the colour histograms of these images by means of Eq. (3). This is carried out for every image of the set.

In Fig. 2, we plot in different ways how the distance between colours behave before (blue) and after (red) performing our colour constancy algorithm in order to know whether our algorithm is able to cope with colour constancy.

Leftmost image shows the distributions of the colour histogram distances, while center and rightmost images are box plots of the distances per image of the set and the whole set of images, respectively. In every box plot, the $75 \%$ and $25 \%$ percentiles describe the limits of a box which has a notch at the $50 \%$ percentile as well as there are lines coming out the box up to the minimum and maximum values.

It is clear that there has been a global reduction in the amount of colour distance between images under different illumination, meaning that our colour constancy algorithm is working properly. The mean distance between colour histograms has been decreased from 0.760 to 0.374 , which is almost a reduction of the $50 \%$. Meanwhile, the standard deviation remains equal if we take all the images (0.157), but is been reduced per image as can be appreciated in Fig. 2. This means the algorithms is not introducing error in transformed images. Finally, we can say that the median has decreased from 0.797 to 0.359 as well.

Finally, in Fig. 3 we show some images obtained using our algorithm. We place the reference image followed by the test image. The third image is the result obtained after transforming the test image. These images provides a seemingly proof of the performance of the colour constancy algorithm.

\section{Conclusions}

The present paper's main aim is to show a procedure based on raw image data that allows, in a framework of coefficient transformations of chromaticity coordinates, to find a colour transformation so that the discrepancy in colour caused by variations in illumination conditions over the scene is greatly reduced. The results of this approach were obtained under a wide range of different illuminations and its performance compared to that of the worst case occurred when no colour constancy is applied. We can state that our algorithm improve colour images and provides a way to stabilise colour in front of illumination changes. 


\section{References}

1. Land, E., McCann, J.: The retinex theory of color vision. Scientific American 6 (1977) 108-129

2. Buchsbaum, G.: A spatial processor model for object colour perception. Journal of Franklin Institute 310 (1980) 1-26

3. Shafer, S.: Using color to separate reflection components. Color Research and Application 10 (1985) 210-218

4. Finlayson, G., Funt, B.: Color constancy with shadows. Perception, Special Issue on the 17th European Conference on Visual Perception 23 (1994) 89-90

5. Funt, B., Drew, M., Ho, J.: Color constancy from mutual reflection. Int. J. Computer Vision 6 (1991) 5-24

6. Finlayson, G., Hordley, S., Hubel, P.: Colour by correlation: A simple, unifying framework for colour constancy. IEEE Trans. on Pattern Analysis and Machine Intelligence 23 (2001) 1209-1221

7. Funt, B., Barnard, K., Martin, L.: Is colour constancy good enough? In: Proc. 5th European Conference Computer Vision. (1998) 445-459

8. Forsyth, D.: A novel algorithm for color constancy. Int. Journal of Computer Vision 5 (1990) 5-36

9. Finlayson, G.: Color in perspective. IEEE Trans. on Pattern Analysis and Machine Intelligence 18 (1996) 1034-1038

10. Finlayson, G., Hordley, S.: Improving gamut mapping color constancy. IEEE Trans. on Image Processing 9 (2000) 1774-1783

11. Brainard, D., Freeman, W.: Bayesian color constancy. J. Opt. Soc. Am. A 14 (1997) 1393-1411

12. Sapiro, G.: Color and illuminant voting. IEEE Trans. on Pattern Analysis and Machine Intelligence 21 (1999) 1210-1215

13. Cardei, V., Funt, B., Barnard, K.: Adaptive illuminant estimation using neural networks. In: Int. Conf. on Artificial Neural Networks. (1998) 749-754

14. Swain, M., Ballard, D.: Indexing via color histograms. In: Proc. Int. Conf. on Computer Vision. (1990) 390-393

15. Press, W., Flannery, B., Teukolsky, S., Vetterling, W.: Numerical Recipes in C: The Art of Scientific Computing. 2on edn. Cambridge University Press (1993) 